\title{
Events with Isolated Leptons and Missing Transverse Momentum in ep Collisions at HERA
}

\author{
Gerhard Brandt*
}

Physical Institute, Philosophenweg 12, 69120 Heidelberg

\begin{abstract}
The analysis of events with isolated leptons and missing transverse momentum in the H1 experiment is discussed for the electron, muon and tau channels. In the Standard Model (SM) framework, production of real $W$-bosons gives rise to such topologies. Contributions to the background are dominated by QCD processes. An excess of observed signal over background presents a chance of the discovery of new physics. The results using the HERA 1994-2006 data set corresponding to $341 \mathrm{pb}^{-1}$ are presented While the $e^{-} p$ sample shows good agreement between data and SM expectation, in $e^{+} p$ collisions an excess over the SM expectation with $3.4 \sigma$ significance is observed at high hadronic transverse momentum.
\end{abstract}

\section{Introduction}

Events with high- $P_{T}$ isolated leptons and missing transverse momentum are sensitive to physics beyond the Standard Model (BSM). Both H1 and ZEUS have previously published searches for events with isolated electrons and muons $[1,2,4,3,5]$ and $\tau$-leptons $[6,7]$. The observation of such events in ep collisions at HERA has created considerable interest in the high-energy physics community (many references can be found in [2]) and is continously updated as new data become available [8, 9, 10]. Section 2 reviews the experimental conditions (collider and detector) of the search, section 3 presents the most important physics processes involved, section 4 presents the search

*email: gbrandt@mail.desy.de 
for $e / \mu+P_{T}^{\text {miss }}$ events, section 5 the search for $\tau+P_{T}^{\text {miss }}$ and section 6 discusses the results and possible interpretations.

\section{Experimental Conditions}

HERA operating at DESY in Hamburg is the worlds only ep collider. It collides electrons or positrons ${ }^{1}$ with protons at a center-of-mass energy $\sqrt{s}=$ $320 \mathrm{GeV}$ for most of its operating time. HERA data taking is divided in two main running periods, HERA-1 in the years 1994 to 2000, and HERA-2 in the years 2000 to 2007. During HERA-1, H1 collected a data set corresponding to $118 \mathrm{pb}^{-1}$ of data, dominated by about $90 \%$ by $e^{+} p$ data. For the HERA2 period, the luminosity has been upgraded and spin rotators installed to provide a longitudinally polarised electron beam. For the results discussed here about $250 \mathrm{pb}^{-1}$ of HERA-2 data have been available to H1. This includes the full $e^{-} p$ data sample which corresponds to a total integrated luminosity of $182 \mathrm{pb}^{-1}$. Data taking continues with $e^{+} p$ running until the end of HERA operation, which is scheduled for June 2007.

Two collider experiments, H1 and ZEUS, operate at HERA. This article focusses on H1, described in detail in [11]. A drawing of the H1 detector is shown in figure 1a. It has almost $4 \pi$ angular coverage to detect missing transverse momentum and the subdetectors are arranged for excellent lepton identification. Track information is provided by central jet chambers, a forward tracker and silicon vertexing detectors. The trackers in a solenoid magnet field allow momentum measurement of charged particles. Energy measurement for electromagnetic and hadronic showers is provided by a LAr (liquid argon) calorimeter in the central and forward (proton beam direction) region, and a lead-fibre spaghetti calorimeter ("SpaCal") in the backward direction. For muon detection, the iron flux return yoke of the magnet is instrumented with limited streamer tubes to measure escaping muons. In the forward direction, there is an additional forward muon detector installed.

To compare recorded data to SM predictions, the relevant physics processes are modelled by Monte Carlo generators. The detector response is simulated by H1 software based on GEANT 3 [12]. The simulated events are then processed through the complete reconstruction and analysis chain, identical to the data treatment.

\footnotetext{
${ }^{1}$ In this article, the term electron refers to both electrons and positrons unless explicitly stated.
} 
a)

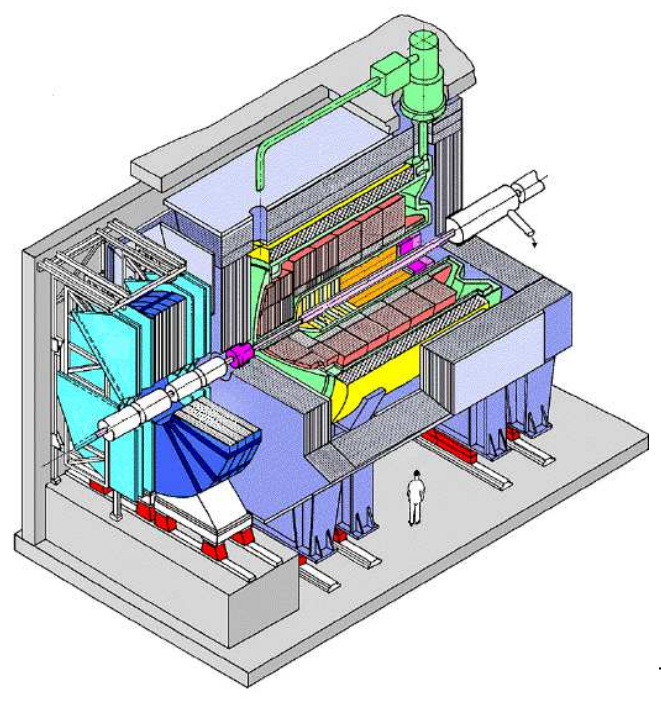

b)

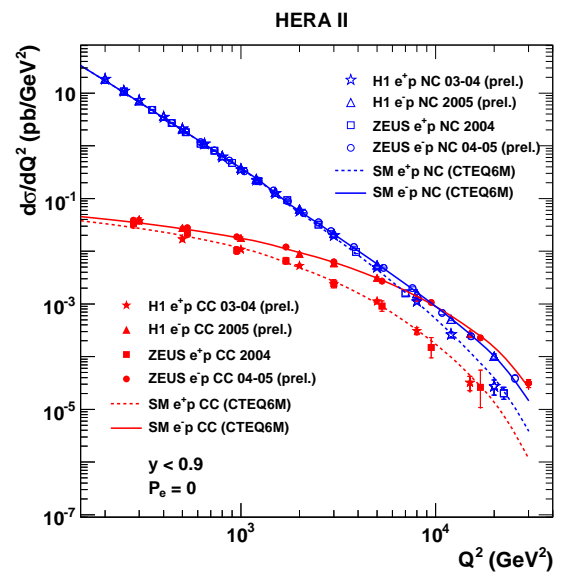

Fig. 1: a) The H1 detector at HERA [11]. Electrons enter from the left side, and protons from the right side; b) A HERA "textbook" measurement: Inclusive unpolarised cross sections of NC and CC DIS processes in ep collisions measured by the $\mathrm{H} 1$ and ZEUS experiments (taken from [13]). The unification of electroweak forces is visible for $Q^{2}>1000$ $\mathrm{GeV}$.

\section{Physics in ep Collisions}

In the Standard Model events with isolated leptons and missing energy are mainly expected from real $W$ production with subsequent leptonic decay,

$$
e p \rightarrow e W^{ \pm}(\hookrightarrow l \nu) X
$$

where $\mathrm{X}$ denotes the hadronic final state (HFS). This is composed of the scattered quark jet and the proton remnant. The main contribution to this process is radiation of a $W$ boson off the scattered quark line, shown in figure $2 \mathrm{a}$. This and other contributing processes are simulated by the EPVEC generator [14]. The total cross section is about $1 \mathrm{pb}$. With a typical detection efficiency of $40 \%$ for the total leptonic branching ratio this means about 100 events are expected in an ep data sample of $250 \mathrm{pb}^{-1}$. For SM $W$ production, the transverse momentum of the HFS $P_{T}^{X}$ is predicted to be well below 25 $\mathrm{GeV}$. At high $P_{T}^{X}$ the SM prediction is low. Looking at its $P_{T}^{X}$ provides a measure for how untypical, or interesting, an event is. An event with a $P_{T}^{X}$ of $29 \mathrm{GeV}$ is shown in figure 3 .

Several physics processes contribute to the background. The most prominent physics processes measured at HERA are neutral current (NC) $e p \rightarrow e X$ 
and charged current (CC) $e p \rightarrow \nu X$ in deep inelastic scattering (DIS), shown in figures $2 \mathrm{~b}$ and $\mathrm{c}$ respectively. These processes are simulated by the RAPGAP [15] and DJANGO [16] generators. The regime of DIS is defined by the negative momentum transfer square $Q^{2}$ of the boson exchanged between the indicent electron and the quark scattered from the proton, at $Q^{2}>1$ $\mathrm{GeV}$. The regime $Q^{2}<1 \mathrm{GeV}$ known as photoproduction is simulated in the PYTHIA [17] framework. Here, two jets are produced by boson-gluon-fusion as shown in figure 2d. For the analysis presented in this paper $\mathrm{NC}$ and $\mathrm{CC}$ in DIS are important background processes due to their high cross sections and event topologies. They are also tools, because they provide high statistics samples of final state objects such as leptons and QCD jets whose properties need to be well understood to make accurate predictions. Figure $1 \mathrm{~b}$ shows measurements of NC and CC cross sections done at HERA.

As a template for physics beyond the Standard Model producing isolated lepton and missing transverse momentum topologies, the anomalous production of top quarks via flavor changing neutral currents (FCNC) is used (shown in Fig. 2e). This process was generated by the ANOTOP [18] programme and used to optimise acceptance for a possible BSM signal.

\section{Search for Events with Isolated Electrons and Muons}

Electrons are detected as isolated, compact electromagnetic clusters in the calorimeter and a high quality track pointing to that cluster. Muons are detected by signals in the muon detectors, matching tracks in the trackers and energy deposits in the LAr calorimeter typical for minimally ionising particles. Electrons and Muons are detected in the phase space of lepton transverse momentum $P_{T}^{l}>10 \mathrm{GeV}$ and polar angular range $5^{\circ} \theta_{l}<140^{\circ}$ $[19,21]$. Leptons are required to be isolated against other tracks and jets in the event. This reduces leptons in or close to QCD jets which are expected to be at lower $P_{T}^{l}$. In DIS the scattered electron is mostly seen in the backward region $\theta_{l}>155^{\circ}$ of the detector. The missing transverse momentum $P_{T}^{\text {miss }}$ is reconstructed from the four-vector sum of all reconstructed final state particles (hadrons and leptons) and required to be greater than $12 \mathrm{GeV}$. This greatly reduces $\mathrm{NC}$ and photoproduction contributions to the background, where $P_{T}^{\text {miss }}$ only appears due to fluctuations in the energy measurement. Additionally SM processes are suppressed topologically, for example by requiring acoplanarity of the lepton and the hadronic final state four-vector in the transverse plane. For neutral current and photoproduction events a back-to-back topology of the final state objects is expected from momentum conservation. 
a)

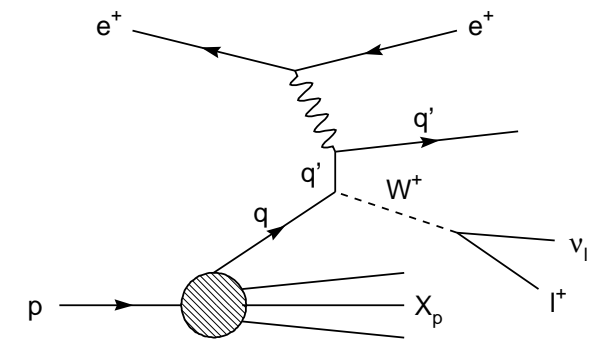

c)

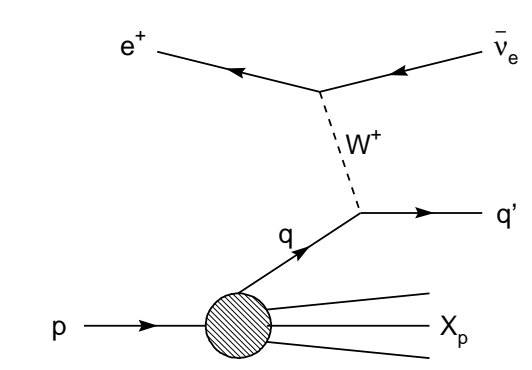

d)

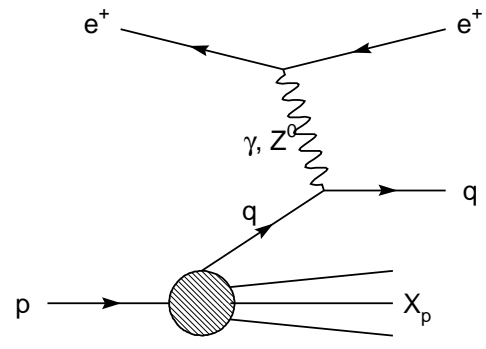

b)

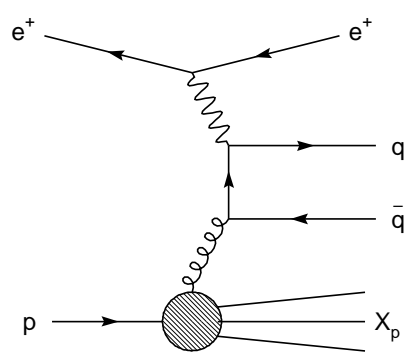

)

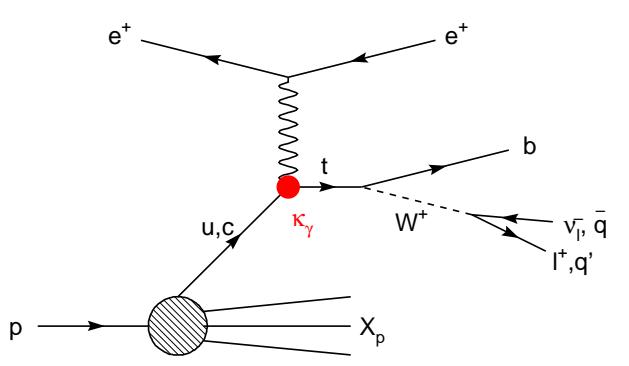

e)

Fig. 2: Physics processes at HERA contributing to $l+P_{T}^{\text {miss }}$ topologies as signal (a and e) or background due to mismeasurements (b,c,d): a) Production of real $W$-Bosons in the Standard Model; b) Neutral current; c) Charged current; d) Photoproduction of jets by boson-gluonfusion; e) Anomalous production of single top quarks via FCNC, the template used for possible BSM contributions. 

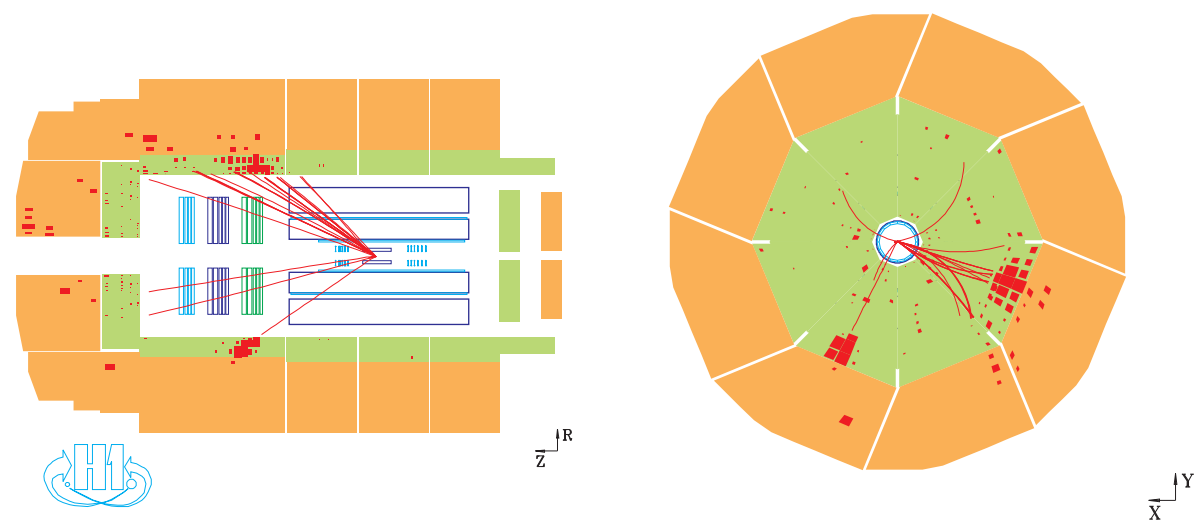

Fig. 3: Event display for an event with an isolated electron of $P_{T}^{e}=37 \mathrm{GeV}$, missing transverse momentum of $P_{T}^{\text {miss }}=44 \mathrm{GeV}$ and a hadronic system of $P_{T}^{X}=29 \mathrm{GeV}$, observed in HERA- $2 e^{+} p$ data. The Standard Model expectation for events with this topology is low.

Figure 4 shows the results of the search for the combined electron and muon channels for the $e^{+} p$ data sample corresponding to $158 \mathrm{pb}^{-1}$ and the $e^{-} p$ data sample corresponding to $184 \mathrm{pb}^{-1}$. A total of 46 events are observed in the complete data, with $43.0 \pm 6.0$ expected from the SM. At large hadronic transverse momentum $P_{T}^{X}>25 \mathrm{GeV}$ a total of 18 events are observed, but only $11.5 \pm 1.8$ predicted. 15 of these events are observed in the $e^{+} p$ sample, where only $4.6 \pm 0.8$ are expected. Only 3 events are seen in the $e^{-} p$ data sample, where $6.9 \pm 1.0$ are expected. The significance of the observed excess in the $e^{+} p$ data sample is $3.4 \sigma$. No excess is observed in the $e^{-} p$ data sample. This observation represents a chance for discovery of new physics at HERA.

\section{Search for Events with Isolated Tau Leptons}

Due to lepton universality in the Standard Model, the tau channel is expected to behave identical to the electron and muon channels. Leptonic $\tau$-decays are included in the electron and muon channels. This search adds hadronic $\tau$ decays with one charged hadron, leading to a 1-prong signature, which covers about $50 \%$ of the total $\tau$-lepton branching ratio [20, 21]. 3-prong decays are not included because the background is too large to yield a significant signalto-background ratio.

Tau-jets are identified based on hadronic jets in the phase space $P_{T}^{\text {jet }}>7$ $\mathrm{GeV}$ and $20^{\circ}<\theta^{\text {jet }}<120^{\circ}$. The candidate jets are expected to be collimated due to the boost of the $\tau$-lepton. This is ensured by requiring a jet radius 


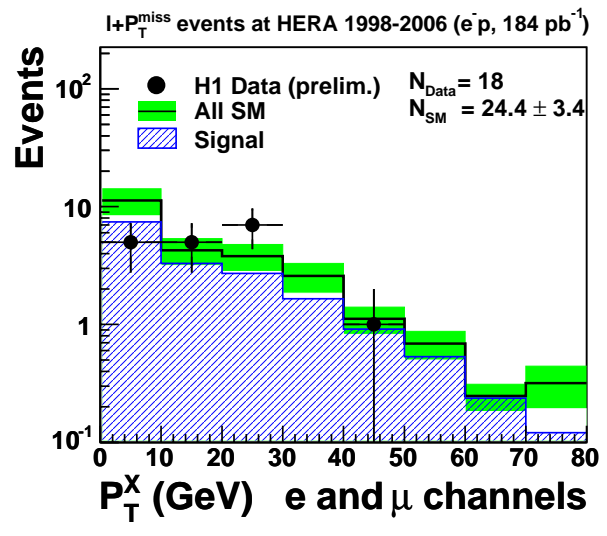

a)

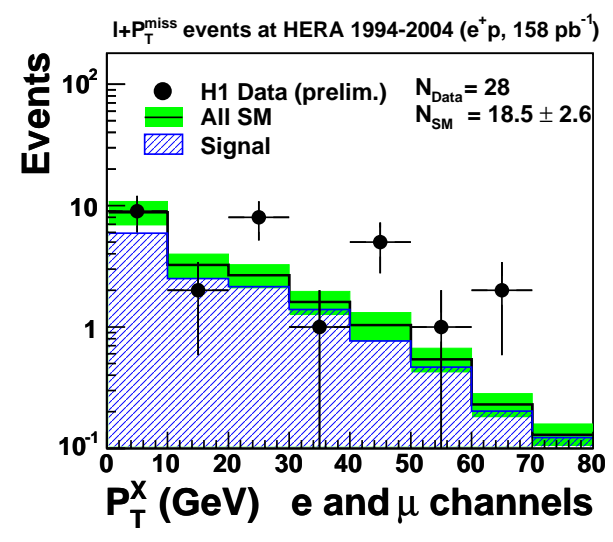

Fig. 4: Transverse momentum of the hadronic system $P_{T}^{X}$ for the combined $e$ and $\mu$-channels in the $e / \mu+P_{T}^{\text {miss }}$ search. The points show the data with statistical errors. The open histogram shows the SM expectation. Systematic and statistical errors added in quadrature are shown as the shaded band. The signal contribution of the SM expectation dominated by real $W$ production is shown as a hatched histogram and amounts to about $70 \%$. While the $e^{-} p$ data (a) agree well with the SM expectation, the $e^{+} p$ data (b) show an excess of events over the SM prediction amounting to $3.4 \sigma$ significance.

$R_{\text {jet }}<0.12$ in the $\eta-\phi$-plane. Exactly one track is required in a cone $D=1.0$ in the $\eta-\phi-$ plane around the jet axis. Neutrinos from the decay chain $W \rightarrow \nu \tau \hookrightarrow l \nu_{l} \bar{\nu}$ are taken into account by requiring $P_{T}^{\operatorname{miss}}>12$ $\mathrm{GeV}$. Because of the similarity of $\tau$-jets to narrow QCD jets with a low track mulitplicity, the signal to background ratio is less favorable than in the electron and muon channels. Jets in photoproduction $\left(Q^{2}<1 \mathrm{GeV}\right)$ may enter the sample due to fake $P_{T}^{\text {miss }}$ from fluctuations in the energy measurement and QCD jets faking $\tau$-jets. $\mathrm{CC}$ background is much higher than in the electron and muon channel. The correct description of dijet $\mathrm{CC}$ events becomes important since their signature is very similar to the events in the interesting region at high PTX. The second jet, which could fake a $\tau$-jet, may come from next to leading order processes or final state radiation.

Figure 5 shows the preliminary results for the $\tau$ analysis. In $e^{+} p$ data, 8 candidates are observed for $10.8 \pm 2.5$ expected. All candidates are in the lowest $P_{T}^{X}$ bin where most of the background is expected. In $e^{-} p$ data 17 events are observed, with $13.5 \pm 2.8$ expected. Three events are observed at $P_{T}^{X}>25 \mathrm{GeV}$, while only $0.74 \pm 0.18$ are expected. At first glance this seems to suggest a trend opposite to the electron and muon channels, but 


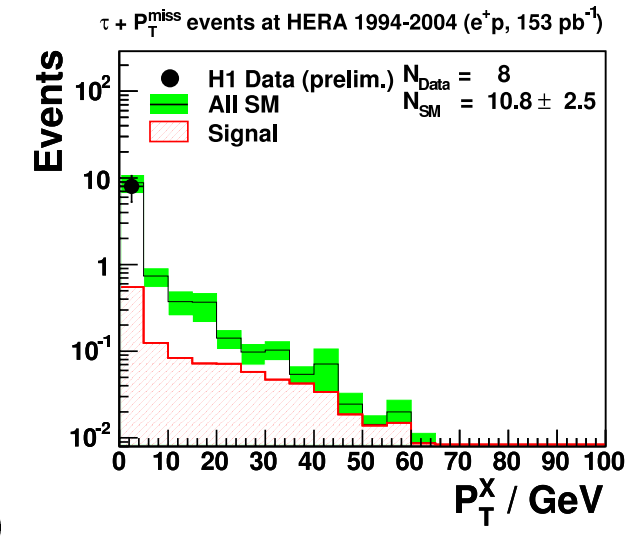

a)

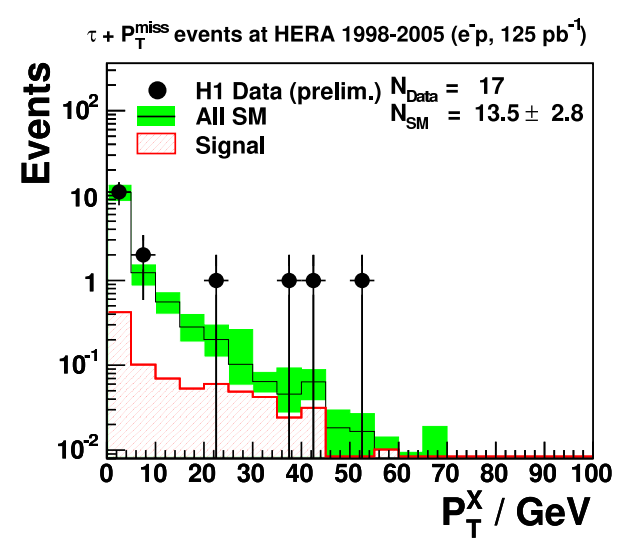

Fig. 5: Transverse momentum of the hadronic system $P_{T}^{X}$ in $\tau+P_{T}^{m i s s}$ events. While there are no candidates at high $P_{T}^{X}$ observed in the $e^{+} p$ sample (a), there are three candidates at high $P_{T}^{X}$ observed in the $e^{-} p$ sample.

this conclusion is premature. The statistical significance of the result is low. Also at closer inspection, some of the three observed candidates seem quite untypical for $\tau$-jets and are more likely background that can possibly be removed in an improved analysis.

\section{Interpretation}

While the low number of observed events in these analyses does not yet show a clear pattern pointing to a strong hypothesis for a BSM process to explain the excess, it is already intriguing to think about possible mechanisms.

Upon the first observation of these events at HERA-1, one of the most exciting interpretations was the production of single top-quarks. While this is allowed kinematically and possible in CC processes at HERA, the cross section is too low to see a signal in the available data samples. If observed, top-quark events could therefore only be produced anomalously via flavor changing neutral currents (FCNC). The process is illustrated in figure $2 \mathrm{e}$ where the scattered quark couples to the exchanged photon (or $Z^{0}$, not shown) via a FCNC coupling and produces a top-quark. The subsequent decay $t \rightarrow b W$ followed by $W \rightarrow l \nu$ or $W \rightarrow q \bar{q}$ produces either the searched $l+P_{T}^{\text {Miss }}$ signature or a 3 jet topology. The $b$-jet gives rise to a highly energetic hadronic system $\mathrm{X}$, giving rise to the high $P_{T}^{X}$. These leptonic and hadronic signatures have been analysed and limits have been set which are competitive to limits on anomalous top production set at other colliders [22].

Another possibility to explain the observation is to explicitly construct 
a model. This could be based on a new particle $N$ with fermion number $\mathrm{F}=0$ coupling to $e-q$. If such a particle had a high mass $m_{N}$ it would have to be produced at large $x_{B j}=M_{N}^{2} / s$. In this region the parton density of valence quarks in the proton is much larger than the density of sea quarks, hence the production cross section for such a particle is much larger in $e^{+} p$ running than in $e^{-} p$ running. In the framework of $R$-parity violating supersymmetry, such particles could be resonantly produced stop-squarks, or sbottom-squarks produced in the t-channel [23]. These preliminary ideas have yet to be intensively studied.

A major issue with the observation is that ZEUS cannot confirm the excess observed by H1, although the analysis is performed in a more restricted phase space. Studies to properly compare the results and test the compatibility of both experiments are ongoing [8].

For the remainder of HERA data taking $e^{+} p$ data will be recorded. If the data taking continues at the current rate, an additional integrated luminosity of $\mathcal{L} \sim 150 \mathrm{pb}^{-1}$ can be expected. At the time of writing the analysis of events with isolated leptons and missing transverse momentum is followed with full effort to extract the maximum of information from this puzzling observation.

\section{References}

[1] T. Ahmed et al. [H1 Collaboration], DESY 94-248 (1994).

[2] C. Adloff et al. [H1 Collaboration], Eur. Phys. J. C 5 (1998) 575 [hep-ex/9806009].

[3] V. Andreev et al. [H1 Collaboration], Phys. Lett. B 561 (2003) 241 [hep-ex/0301030].

[4] J. Breitweg et al. [ZEUS Collaboration], Phys. Lett. B 471 (2000) 411 [hepex/9907023].

[5] S. Chekanov et al. [ZEUS Collaboration], Phys. Lett. B 559 (2003) 153 [hepex/0302010].

[6] S. Chekanov et al. [ZEUS Collaboration], Phys. Lett. B 583 (2004) 41 [hepex/0311028].

[7] A. Aktas et al. [H1 Collaboration], submitted to Eur. Phys. J. C [hep-ex/0604022].

[8] H1 Collaboration, H1prelim-06-162 ${ }^{3}$.

[9] ZEUS Collaboration, ZEUS-prel-06-012 ${ }^{4}$.

[10] H1Collaboration, H1prelim-06-064 ${ }^{3}$.

[11] H1 Collaboration, The H1-Detector at HERA, Nucl. Instr. and Meth. A 386 (1997) 310 and 348 . 
[12] GEANT 3, R. Brun et al., CERN-DD/EE/84-1.

[13] H1 Collaboration, H1prelim-06-041 ${ }^{3}$;

B. Portheault, Dissertation, Université Paris-Sud XI $(2005)^{5}$.

[14] U. Baur, J. A. M. Vermaseren and D. Zeppenfeld, Nucl. Phys. B 375 (1992) 3.

[15] H. Jung, Comput. Phys. Commun. 86 (1995) 147, RAPGAP program manual (1998) http://www-h1. desy.de/ jung/RAPGAP.html.

[16] G. A. Schüler and H. Spiesberger, DJANGO 2.1, Proc. of the Workshop "Physics at HERA" (1991), Eds. W. Buchmüller and G. Ingelman., Vol. 3, p. 1419.

[17] T. Sjöstrand, PYTHIA 5.7, Comput. Phys. Commun. 82 (1994) 74.

[18] E. Perez, ANOTOP, unpublished.

[19] D. South, Dissertation, University of Manchester (2003) ${ }^{5}$.

[20] C. Veelken, Dissertation, University of Liverpool (2006) ${ }^{5}$.

[21] G. Brandt, Dissertation, University of Heidelberg (2007, in preparation) ${ }^{5}$.

[22] A. Aktas et al. [H1Collaboration], Eur. Phys. J C 33 (2004) 9 [hep-ex/0310032].

[23] E. Perez, private communication For more information on (R-parity violating) SUSY try:

J. Haller, Dissertation, University of Heidelberg $(2003)^{5}$

S. Martin, A Supersymmetry Primer, [hep-ph/9709356].

\footnotetext{
${ }^{3} \mathrm{H} 1$ preliminary results available at: http://www-h1.desy.de/publications/H1preliminary.short_list.html

${ }^{4}$ ZEUS preliminary results available at: http://www-zeus.desy.de

${ }^{5} \mathrm{H} 1$ theses available at:

http://www-h1.desy.de/publications/theses_list.html
} 